\title{
The ability of the saccadic system to change motor plans in scanning letter strings
}

\author{
DORINE VERGILINO-PEREZ and CÉCILE BEAUVILLAIN \\ Université René Descartes, Boulogne Billancourt, France
}

\begin{abstract}
Evidence from recent studies bolsters the idea of preestablished motor plans in scanning isolated items. Thus, refixation saccades are preplanned at the same time as the primary saccade directed to a peripheral item and are completed with fixed amplitudes irrespective of the first fixation position in the item. In order to examine the saccadic system's ability to correct the motor plan during its execution on the basis of new visual information, an experiment was conducted in which 11-letter strings were changed to two 5-letter strings at different times after the primary saccade was directed to the stimulus. The results demonstrate that the saccadic system is able to cancel the preplanned refixation saccade and plan a saccade directed to the next item only when the new visual information is available at least $220 \mathrm{msec}$ before the execution of the saccade.
\end{abstract}

It is well known that low-level visual information, such as the spaces between words and the length of a to-befixated word, is the major determinant of where the eyes initially land in a word (McConkie, Kerr, Reddix, \& Zola, 1988; O'Regan, 1979; Rayner, 1979). Low-level information also influences whether a refixation saccade is made on the word ${ }^{1}$ (McConkie, Kerr, Reddix, Zola, \& Jacobs, 1989; O’Regan, Lévy-Schoen, Pynte, \& Brugaillère, 1984) and where the saccade lands (Beauvillain, Dukic, \& Vergilino, 2000). Recently, Reichle, Pollatsek, Fisher, and Rayner (1998) have proposed that a refixation saccade is automatically planned after the initial saccade is completed. However, studies in which isolated words were read have shown that the saccadic system can acquire length information in periphery in order to plan more than one saccade at a time. A preestablished organized plan has been proposed for the refixation saccade that could constitute a unit of motor action encoded with the primary saccade and memorized before the execution of the last one (Beauvillain et al., 2000; Vergilino \& Beauvillain, $2000,2001)$. However, the initial representation of the refixation saccade may be canceled during the first fixation on the word. How much time the saccadic system needs to cancel the initial refixation plan and to redirect the second saccade toward the next word is still unknown.

In the present experiment, we investigated the scanning of nonsense strings rather than words in order to eliminate possible influences of nonperceptual factors such as meaning or familiarity. We used an eye-movement-contingent display change in which a single long letter string was seg-

We thank Simon P. Liversedge, George McConkie, and an anonymous reviewer for their helpful comments on an earlier version of the manuscript. Requests for reprints should be addressed to D. Vergilino-Perez, Laboratoire de Psychologie Expérimentale, UMR 8581 CNRS, Université René Descartes, 71 Avenue Edouard Vaillant, 92774 Boulogne Billancourt Cedex, France (e-mail: vergilino@psycho.univ-paris5.fr). mented into two short letter strings at different times during the initial fixation on the item. Thus, observers had to cancel an initial plan to refixate a long letter string and program a saccade directed to the second, short item. In this situation, the initial spatial representation used for planning saccadic eye movement had to be completely transformed. Indeed, we previously showed a different coding of the interword and refixation saccades in reading isolated words (Vergilino \& Beauvillain, 2001). When the second saccade is directed within the same word, the linear regression of the second saccade position on the first position has a slope close to 1 . This indicates that such a saccade is coded as a fixed-size motor vector determined by word length, irrespective of the initial landing position in the item. Clearly, such a saccade does not aim for a precise target position in the word, whereas the saccade directed toward a new word selected in periphery tends to aim for a target position left of the center of the selected word. If the eyes always land precisely at the center of the second word regardless of the position of the current fixation, we predict a slope of 0 between the two successive interword saccades. However, replicating data observed previously in reading text (McConkie et al., 1988), we found a slope near 0.5 . This reflects the fact that the interword saccade, influenced by its launch site, lands on the center of gravity of the configuration between the current fixation and the end boundary of the selected word. Altogether, our data showed that refixation saccades are coded in an oculocentric coordinate system as a constant movement of the eye in the orbit, whereas interword saccades are coded in a retinocentric representation to aim for a precise part of the word.

The question of interest here is to determine the time required for the saccadic system to cancel an initial plan to refixate a long letter string and program a saccade directed to another item. The present experiment bears a close resemblance to those of many studies using a par- 
adigm in which changes are made in the visual display, contingent on the preparation or execution of the movement (Becker \& Jürgens, 1979; McConkie \& Rayner, 1975). Such a paradigm has provided important evidence of the understanding of the capacity of the saccadic system to change previous motor plans. In a previous experiment, we examined the flexibility of the saccadic system to correct the amplitude of the refixation saccade in scanning isolated items (Vergilino \& Beauvillain, 2000). The length of long nonsense letter strings was increased or decreased by adding or subtracting two letters at the end of the item, either at the time of the first saccade or at various points in time thereafter. We demonstrated that the saccadic system could correct the refixation saccade motor plan relative to the new length if the change occurred at least $150 \mathrm{msec}$ before the execution of the refixation saccade.

What are the implications of such results for the present experiment? A simple correction of the refixation saccade vector takes at least $150 \mathrm{msec}$; a greater delay is expected when the change of the saccadic motor plan involves the cancelation of the refixation saccade. In this case, the saccadic system must select a new target position in the second item and program the saccade directed to this target position. The experiment presented here was concerned with the response of the saccadic system to a segmentation of one 11-letter string into two 5-letter strings by substitution of the sixth letter with a blank space at different times $(0,50,140$, and $220 \mathrm{msec})$ during the initial fixation reckoning from the end of the primary saccade. The initial letter string length was chosen in order to trigger a refixation saccade, since a previous experiment has shown that the probability of refixating isolated 11-letter strings is quite high ( $90 \%$; Vergilino \& Beauvillain, 2000). As was found in preliminary tests, the amplitude of the saccade directed to the second 5letter string is expected to be about twice the amplitude of the refixation saccade directed to a second position in the 11-letter string. Consequently, the modification of the motor plan should be apparent from the relation between the amplitude of the second saccade and the delay between the stimulus change and the triggering of the second saccade. The greater the delay until the change, the greater is the possibility that the saccadic system should modify the initial plan to refixate the stimulus into a saccade directed toward the new second string of letters. Two control conditions, which consisted of 11-letter strings and two 5-letter strings with no stimulus change, were added.

\section{METHOD}

\section{Subjects}

Six students were paid to participate in the experiment. All were native French speakers with normal uncorrected vision. They were unfamiliar with the purpose of the experiment.

\section{Procedure}

The subjects were tested individually. They sat in an adjustable chair, and their heads were restrained with a submaxillar dental print and a forehead rest. Each session began with calibration of the eye-tracking system. In each session, the subjects were required to sequentially fixate five positions along a horizontal line of $12^{\circ}$ angle. To check the accuracy of the eye movement recording with respect to the last calibration procedure performed, each experimental trial was preceded and followed by fixation bars $6^{\circ}$ to the left and to the right of the screen center (Figure 1).

The sequence of events during an experimental trial was as follows: (1) The subjects had to fixate a calibration bar displayed $6^{\circ}$ to the left of the screen center. (2) When the computer detected an accurate fixation on the bar, a fixation cross replaced the bar. The subjects were required to remain fixated on the cross until it disappeared. (3) The disappearance of the fixation cross was the signal to move the eyes toward the stimulus. If the computer detected a saccade before the fixation cross disappeared, the trial was discarded. At the time when the cross disappeared, the target letter string was displayed at a visual angle of $1^{\circ} 30^{\prime}$, corresponding to three character spaces to the right of the fixation cross, followed by

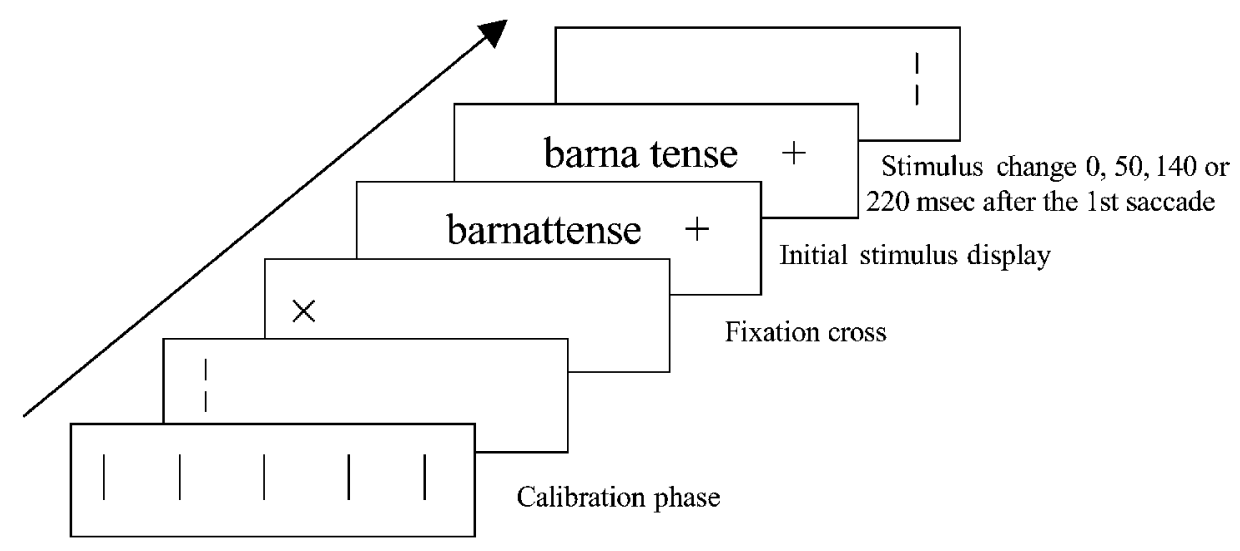

Figure 1. Procedure employed in the experiment. After a calibration phase, the subjects fixated a cross and, at its disappearance, the stimuli were displayed at a visual angle of $1.30^{\circ}$ to the right of the cross. The 11-letter strings could be transformed into two 5 -letter strings at different times $(0$, 50,140 , or $220 \mathrm{msec}$ ) during the initial fixation reckoning from the end of the primary saccade. 
a cross displayed three character spaces from the end of the target letter string; the subjects had to scan the target letter string. The 11letter string could be transformed into two 5-letter strings at different times $(0,50,140$, or $220 \mathrm{msec})$ during the initial fixation reckoning from the end of the primary saccade. (4) After scanning the items, the subjects directed their eyes toward the second cross and pressed a button; when the button was pressed, the stimuli were erased and a fixation bar was displayed $6^{\circ}$ to the right of the screen center.

The subjects received 40 practice trials with the same characteristics as the test trials.

\section{Design}

A single-factor design was used, in which the delay of the stimulus change $(0,50,140$, or $220 \mathrm{msec}$ after the onset of fixation) was a within-subjects factor. Two control conditions with no stimulus change were added. The first control condition consisted of 11-letter strings, and the second, of two 5-letter strings. Each subject was exposed to the six conditions, but no subject saw the same item more than once. Nevertheless, each item was presented under the six conditions across subjects. With the aim of preventing the subjects from expecting an item change during each trial, we introduced two filler conditions, yielding equal numbers of change and no-change trials. In order to reduce the visual difference between fillers and experimental trials, the lengths of the fillers-13-letter items or 3letter items followed by 7-letter items-were close to the lengths of the experimental items. All conditions were mixed within the different experimental sessions, and the items were presented in random order.

\section{Materials}

Two hundred ten 11-letter nonsense strings were constructed so that each item could be divided into two 5-letter items by removing the 6th letter. The items were constructed in such a way that the removal of the 6th letter did not modify the phonological coding of the item (e.g., barnattense/barna tense). The initial 11-letter and final 5-letter strings adhered to the orthographic and phonological rules of French. We selected 35 frequent initial bigrams in French and constructed eighteen 11-letter strings from each bigram. We also constructed 105 fillers with the same characteristics as the experimental letter strings. Each subject performed 840 trials in three separate experimental sessions of 280 trials each. Each experimental session contained 210 experimental trials and 70 filler trials.

\section{Apparatus}

The stimuli were presented on a Hewlett-Packard 1310A CRT (P15 phosphor) display interfaced with a fast graphic system providing a frame frequency of $1000 \mathrm{~Hz}$. One character subtended $30 \mathrm{~min}$ of visual angle. Eye movements were monitored using a Dr. Bouis oculometer system. The eye tracker had an absolute resolution of $6 \mathrm{~min}$ of arc, and the output was linear over $12^{\circ}$ of visual angle. The signal from the oculometer was sampled every $2 \mathrm{msec}$. Complete details of the eye-movement-recording apparatus, calibration procedure, and numerical data processing can be found in Beauvillain and Beauvillain (1995). Primary saccade starting and ending points were calculated using a velocity criterion of $25^{\circ} / \mathrm{sec}$.

\section{RESULTS}

We excluded $5 \%$ of the data from the analyses because of a lack of accuracy in the eye position or because of the occurrence of blinks or regressive saccades. Trials in which the subject made a regression (1\% of total trials) or only one fixation on the letter string (3\% of total trials) were also removed from the analyses. Subsequent analyses were performed on the remaining trials, including $85 \%$ of two-fixation cases and $6 \%$ of three-fixation cases.

\section{Second Saccade Amplitude}

As was expected, second saccade amplitude, calculated in character spaces, was larger in the 5-letter control condition $(M=5.4)$ than in the 11-letter control condition $[M=3.9 ; F(1,5)=301.38, p<.0005]$. The time of the stimulus change affected the second saccade amplitude $[F(3,15)=22.87, p<.0005]$. When the stimulus change occurred during the primary saccade and $50 \mathrm{msec}$ after the first fixation onset, intermediate saccade amplitudes were obtained $(M \mathrm{~s}=5$ and 4.3 , respectively). They differed from the 11-letter $[F(1,5)=65.86, p<.0005$, and $F(1,5)=34.16, p<.002$ for the 0 - and 50-msec delays, respectively $]$ and 5-letter $[F(1,5)=9.95, p<.02$, and $F(1,5)=51.44, p<.001$ for the 0 - and 50-msec delays, respectively] control conditions. When the stimulus change occurred 140 and $220 \mathrm{msec}$ after the first fixation onset, saccade amplitude was similar to that obtained in the 11-letter control condition $[M \mathrm{~s}=3.9$ and 3.5 , respectively; $F<1$ and $F(1,5)=3.96, p<.10$, respectively] and different from that obtained in the 5-letter condition $[F(1,5)=87.52$ and $F(1,5)=67.88$, respectively; $p$ s $<.0005]$.

Nevertheless, this global analysis does not take into account the delay between the display change and the triggering of the refixation saccade that is expected to influence the modification of the saccade amplitude calculation. Since first fixation duration for change conditions did not differ from that of control conditions ( $M$ of 367 and $363 \mathrm{msec}$, respectively; $F<1$ ), the delay was evenly distributed from $0 \mathrm{msec}$ to $500 \mathrm{msec}$. Interestingly, the modification of the second saccade amplitude in the 0 - and 50-msec delay conditions was not accompanied by an increase in the first fixation duration $[F(1,5)=$ 2.28 , n.s.].

Second saccade amplitude was examined as a function of the delay between the stimulus change and the triggering of the saccade (Figure 2). Linear regressions were performed by the least squares fit procedure to describe the relation between the two parameters: delay and second saccade amplitude. Two different linear relations of saccade amplitude versus delay can be seen. In order to clarify the limits of these two relations, we performed a statistical analysis using the following procedure. The linear least square fits were calculated by varying the limits, $D_{\min }$ and $D_{\max }$ (where $\mathrm{D}$ is delay). $\mathrm{D}_{\min }$ was first fixed to the negative minimum value of the delay, and the variation of the slope of the linear function versus $\mathrm{D}_{\max }$ was computed. This variation exhibits a breaking point $\left(d_{1}\right)$, considered as the limit of the first linear fit $(220 \mathrm{msec})$. The same procedure was applied to the second linear fit obtained by fixating $\mathrm{D}_{\max }$ to the maximum value of $\mathrm{D}$ and varying $\mathrm{D}_{\min }$. The breaking point of this second linear fit $\left(d_{2}\right)$ was $300 \mathrm{msec}$. These two different breaking points revealed the limits of two different classes 


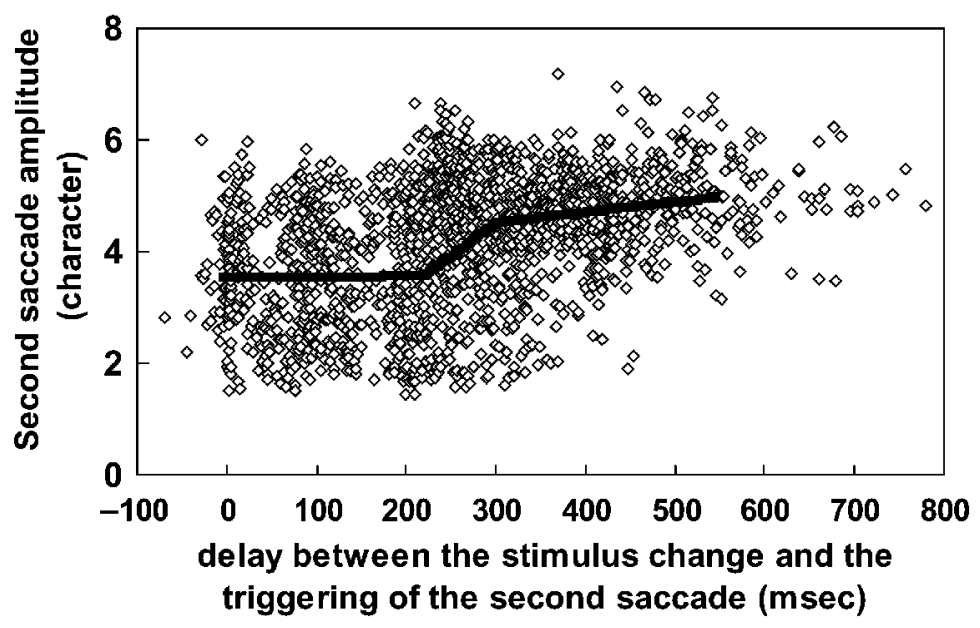

Figure 2. Amplitude of the second saccade as a function of the delay between the stimulus change and the triggering of the second saccade in the 11-to-5letter string condition. Negative values correspond to second saccades triggered before the stimulus change, whereas positive values correspond to second saccades triggered after the stimulus change.

of saccade amplitudes. An arbitrary slope was plotted between $d_{1}$ and $d_{2}$. For saccades triggered less than $220 \mathrm{msec}$ before or more than $300 \mathrm{msec}$ after the stimulus change, the equations of the linear regressions are $y=.0001 x+3.5$ and $y=.002 x+4$, respectively. The value of the $b$ parameter of the linear function indicates that for saccades triggered less than $220 \mathrm{msec}$ after the stimulus change, the amplitude $(b=3.5)$ is very similar to that obtained for refixation saccades in the 11-letter control condition $(M=3.9)$. Thus, the increase of the saccade amplitude to reach the second word occurs 220-300 msec after the stimulus change. After $300 \mathrm{msec}$, the slightly positive slope of the linear function suggests that the saccade continues to increase with the delay.

The relation between the second saccade amplitude and the delay was examined individually in order to find the breaking points for the six subjects. Two mean breaking points-of $228 \mathrm{msec}$ and $316 \mathrm{msec}$ after the stimulus change-were obtained, with a significant difference between them $[F(1,5)=17.90, p<.008]$. Moreover, the second saccade amplitude triggered before the first breaking point differed from that obtained between the two breaking points [ $M$ s of 3.39 and 4.47 character spaces; $F(1,5)=11.65, p<.01]$. Second saccade amplitude triggered after the second breaking point ( $M$ of 4.87 character spaces) also significantly differed from that obtained between the two breaking points $[F(1,5)=12.38$, $p<.01]$.

\section{Relation Between the First and the Second Fixation Positions}

To estimate whether the modification of the saccade amplitude entailed a complete transformation of the initial refixation saccade into a saccade directed to the sec- ond letter string, it is important to examine the relationship between the first and the second fixation positions. Indeed, the slope of the linear function should be close to 1 for second saccades triggered before the modification of the saccade amplitude if such initial saccades are planned as fixed motor vectors refixating the initial string regardless of the first fixation position into it. Alternatively, the slope should be close to 0.5 for second saccades triggered after the modification of the saccade amplitude. This is because, in this case, the saccade is updated relative to the first fixation position in order to aim for a target location corresponding to the center of gravity between the launch site of the saccade and the end of the second 5-letter item (Vergilino \& Beauvillain, 2001).

In Figure 3, the relation between the first and the second fixations is plotted both for control conditions (panel A) and for saccades triggered before the first breaking point (panel B, left) and after the second breaking point (panel B, right) in the 11-to-5-letter conditions. The slope of 0.56 obtained in the 5-letter control condition significantly differed from the slope of 1.18 obtained in the 11-letter control condition $[F(1,5)=8.94, p<.03]$. Moreover, the slope of 1.35 obtained for saccades triggered before the correction of the motor plan significantly differed from the slope of 0.61 obtained for saccades triggered after the correction $[F(1,5)=11.61, p<.01]$. For saccades triggered before the correction of the motor plan, the slope close to 1 indicates a mechanism that encodes the second saccade as a fixed-motor vector, irrespective of the first fixation position. The relationship between the first and the second saccades is similar to that obtained for the 11-letter control condition but differs from that obtained for the 5-letter control condition $[F(1,5)=$ 1.80 , n.s., and $F(1,5)=7.60, p<.03$, respectively]. For 


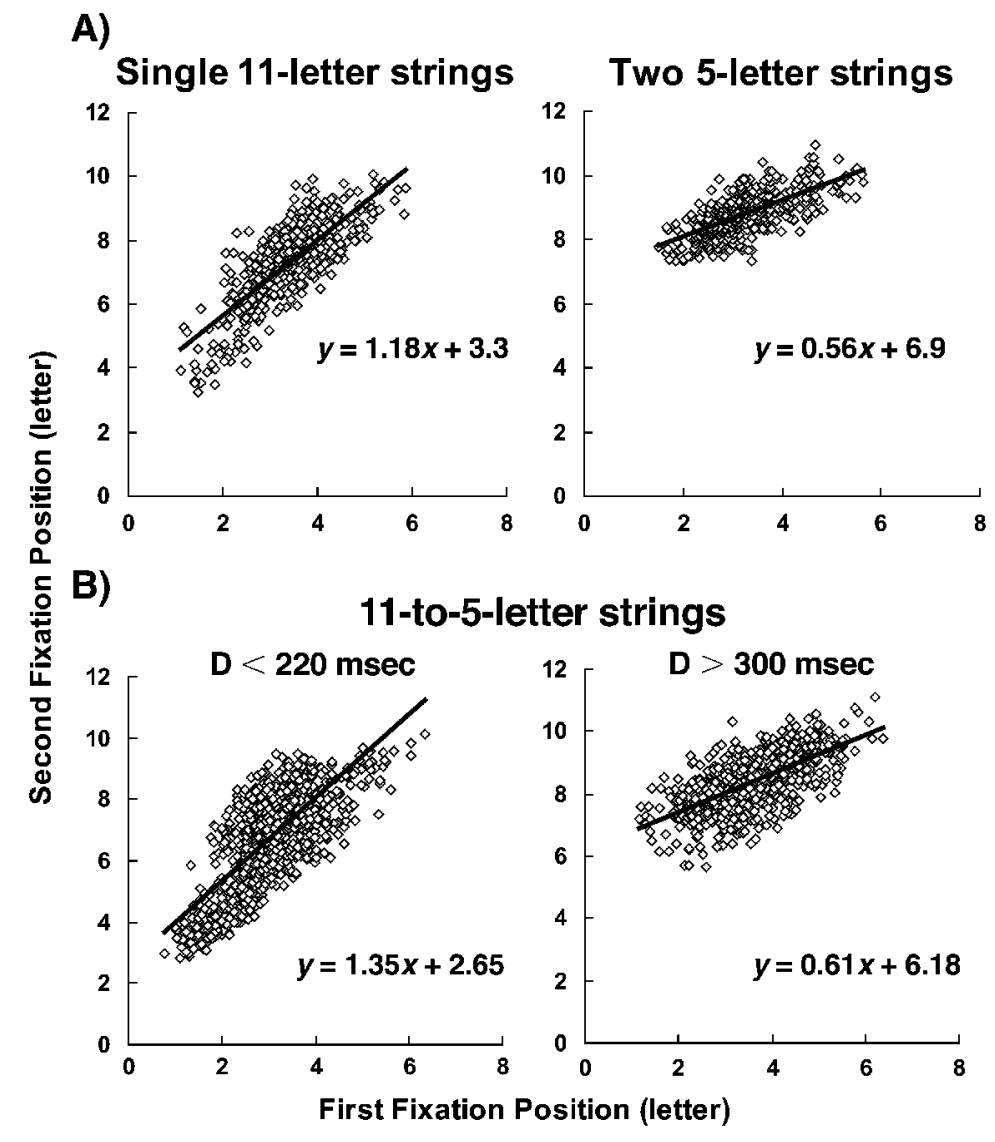

Figure 3. Second fixation positions plotted as a function of first fixation positions for second saccades $(A)$ in the control conditions and (B) triggered before and after the modification of the motor plan in the 11-to-5-letter string condition. The 7 th letter of each 11-letter string corresponds to the 1st letter of the second 5-letter string.

saccades triggered more than $300 \mathrm{msec}$ after the stimulus change, the slope close to 0.5 is similar to that obtained for the 5-letter control condition but differs from that obtained for the 11-letter control condition $[F<1$ and $F(1,5)=14.37$, respectively; $p$ s $<.01]$. This indicates the presence of an updating mechanism of the second saccade, computed to aim for the center of gravity between the launch site of the saccade and the end of the second item.

\section{DISCUSSION}

The present experiment yields new information about the response time of the saccadic system to a change in letter string boundary information. During the execution of a two-saccade sequence, the modification of a long letter string into two short letter strings at different times after the primary saccade leads to a complete updating of the second saccade calculation. The time involved in processing the new boundary information and in recalculating the second saccade is at least $220 \mathrm{msec}$. Saccades that occur sooner are not affected by the stimulus change and are refixation movements encoded as fixed motor vectors with no target position. A saccade that occurs later is directed to the new second item and updated relative to the eye position after the first saccade, to aim for a target position located at the center of gravity between the launch site of the saccade and the end of the second item. The response time estimation of the saccadic system is longer than that obtained in a previous experiment, in which we investigated the adjustment of the refixation saccade amplitude to a change in item length (Vergilino \& Beauvillain, 2000). In that experiment, the length change induced a modification of the ongoing program, requiring simply an adjustment of the refixation motor vector to the new length information. In this case, we found a minimum reprocessing time of about $150 \mathrm{msec}$. In the experiment presented here, the stimulus change involved an interruption of the refixation ongoing program followed by the planning of a saccade directed to another item. We assume that the difference in the minimum reprocessing time results from the fact that the change of the motor 
plan involves the transformation of the spatial information from an oculocentric representation to a retinotopic one. The former specifies a fixed amplitude to cover a distance over the item, and the latter, a target location with respect to the current eye position. Such a representation requires access to an accurate estimate of the new target position as a function of the current eye position.

Overall, the results of these two experiments provide us with insight into the temporal events linked to the refixation saccade execution in reading isolated items. Notably, one implication of this finding is that the refixation saccade amplitude can be modified, whereas the decision to execute the motor preprogram of the saccade is irrevocable. The final decision regarding execution of the preplanned refixation saccade must be made at least $220 \mathrm{msec}$ before the initiation of the saccade-that is, early during the first fixation. However, the vector motor of the saccade can be modified up to $150 \mathrm{msec}$ before it is triggered.

In this study, we have manipulated the spatial information related to where items begin and end. Such information being the main determinant of the landing positions of saccades in reading, we think that we can extend the present results concerning the time required for the saccadic system to change the motor plan to the normal reading situation, even if the decision to cancel a refixation saccade in reading may be taken on higher level influences. However, the present study suggests that the selection of a new target word for the saccadic system occurs early during the first fixation on a word.

\section{REFERENCES}

BEAUVILlain, C. (1996). The integration of morphological and wholeword form information during eye fixations on prefixed and suffixed words. Journal of Memory \& Language, 35, 801-820.

Beauvillain, C., \& Beauvillain, P. (1995). Calibration of an eye movement system for use in reading. Behavior Research Methods, Instruments, \& Computers, 27, 331-337.

Beauvillain, C., Dukic, T., \& Vergilino, D. (2000). Planning twosaccade sequences in reading. In A. Kennedy, R. Radach, D. Heller,
\& J. Pynte (Eds.), Reading as a perceptual process (pp. 327-354). Oxford: Elsevier.

BECKER, W., \& JÜRGENS, R. (1979). An analysis of the saccadic system by means of double step stimuli. Vision Research, 19, 967-983.

McConkie, G. W., Kerr, P. W., Reddix, M. D., \& Zola, D. (1988). Eye movement control during reading: I. The location of initial eye fixations on words. Vision Research, 28, 1107-1118.

McConkie, G. W., KeRr, P. W., Reddix, M. D., Zola, D., \& JaCobs, A. M. (1989). Eye movement control during reading: II. Frequency of refixating a word. Perception \& Psychophysics, 46, 245-253.

McConkie, G. W., \& RAYNER, K. (1975). The span of the effective stimulus during a fixation in reading. Perception \& Psychophysics, 17, 578-586.

O'REGAN, [J.] K. (1979). Saccade size control in reading: Evidence for the linguistic control hypothesis. Perception \& Psychophysics, 25, 501-509.

O'Regan, J. K., Lévy-Schoen, A., Pynte, J., \& Brugaillère, B. (1984). Convenient fixation location within isolated words of different length and structure. Journal of Experimental Psychology: Human Perception \& Performance, 10, 250-257.

PYNTE, J. (1996). Lexical control of within-word eye movements. Journal of Experimental Psychology: Human Perception \& Performance, 22, 958-969.

RAYNER, K. (1979). Eye guidance in reading: Fixation locations within words. Perception, 8, 21-30.

Reichle, E. D., Pollatse K, A., Fisher, D. L., \& Rayner, K. (1998). Toward a model of eye movement control in reading. Psychological Review, 105, 125-157.

Vergilino, D., \& Beauvillain, C. (2000). The planning of refixation saccades in reading. Vision Research, 40, 3527-3538.

Vergilino, D., \& Beauvillain, C. (2001). Reference frames in reading: Evidence from visually and memory-guided saccades. Vision Research, 41, 3547-3557.

VITU, F. (1991). The influence of parafoveal preprocessing and linguistic context on the optimal landing position effect. Perception \& Psychophysics, 50, 58-75.

\section{NOTE}

1. Linguistic factors such as word frequency (McConkie et al., 1988; Vitu, 1991) and morphemic information integrated during the first fixation (Beauvillain, 1996; Pynte, 1996) influence the probability of refixating a word only slightly.

(Manuscript received April 5, 2002; revision accepted for publication March 5, 2003.) 